\title{
Classes of Strongly Stabilizing Bandpass Controllers for Flexible Structures
}

\author{
Alberto Cavallo, Giuseppe De Maria, Ciro Natale, and Salvatore Pirozzi \\ Dipartimento di Ingegneria dell'Informazione, Seconda Università degli Studi di Napoli, Via Roma 29, 81031 Aversa, Italy \\ Correspondence should be addressed to Alberto Cavallo, alberto.cavallo@unina2.it
}

Received 6 August 2011; Accepted 9 October 2011

Academic Editor: Emil Manoach

Copyright (๑) 2012 Alberto Cavallo et al. This is an open access article distributed under the Creative Commons Attribution License, which permits unrestricted use, distribution, and reproduction in any medium, provided the original work is properly cited.

This paper proposes different design strategies of robust controllers for high-order plants. The design is tailored on the structure of the equations resulting from modeling flexible structures by using modal coordinates. Moreover, the control laws have some characteristics which make them specially suited for active vibration reduction, such as strong stabilization property and bandpass frequency shape. The approach is also targeted the case of more sensors than actuators, which is very frequent in practical applications. Indeed, actuators are often rather heavy and bulky, while small and light sensors may be placed more freely. In such cases, sensors can be usefully placed in the locations where the primary force fields act on the structure, so as to provide the controller with a direct information on the disturbance effects in terms of structural vibrations. Eventually, this approach may lead to uncolocated control strategies. The design problem is here solved by resorting to a Linear Matrix Inequality technique, which allows also to select the performance weights based on different design requirements, for example, a suitable bandpass frequency shape. Experimental results are presented for a vibration reduction problem of a stiffened aeronautical panel controlled by piezoelectric actuators.

\section{Introduction}

Reduction of vibrations in flexible systems has been considered for years a challenging problem, due to some specific aspects. First, as the model of the structure is described by partial differential equations that can be treated as an infinite set of ordinary differential equations, any finite-order controller must rely on a limited (truncated) description of the plant. Thus, neglected dynamics actions must be taken into account by robustness properties of the controller, otherwise, spillover effects [1] may lead to control problems, even to the instability of the closed-loop system, in the most unfavorable situation. A second problem is that, in order to base the design technique for the controller on a meaningful model of the structure, many structural modes must be included in the model, especially for structures with high modal density, so that control problems for flexible structures are intrinsically large-scale problems. Only in the simpler case of low modal density nonoptimal control strategies (e.g., sliding manifold-based control [2] may be used). Finally, stability of the controller (in order to reduce sensitivity to disturbances) and limited bandwidth, for example, to avoid spillover, due to interaction of unmodelled dynamics with control action, must be enforced. From a practical point of view, vibrations are often measured by using accelerometers, which are prone to offsets which can even saturate actuators if not suitably filtered out in the feedback loop. Thus, the need for a filtering action both at high and at low frequency arises. Hence, a limited-bandwidth strongly stabilizing controller is needed. Further discussions on this topic can be found in [3, 4]. Furthermore, in [5] a broader list of references on the topic is presented and discussed.

In this paper the selection of a stable stabilizing controller with bandpass frequency shape is discussed. First, optimal controllers are discussed, with analytical expression in closed form that is useful when high-order models may result into increased computational burden. The main limitation of this approach is that it can deal only with square plants, that is, as many inputs as outputs must be present. Next, a further 
technique, able to deal with rectangular plants (i.e., with more outputs than inputs), is proposed. The motivation is provided by the requirements of many applications, where the rejection of a disturbance acting on the structure is desired in locations where the installation of actuator/sensor couples is prohibited. Therefore, the possibility to exploit the vibration measurement in those locations appears the only viable option. The second control strategy proposed in this paper is specifically designed to usefully exploit the knowledge of the disturbance entry point on the structure to place additional sensors able to provide the controller with the necessary information on the vibration status caused by the disturbance. The resulting controller thus has more inputs than outputs, but it is still guaranteed to be strongly stabilizing and with bandpass frequency response. Moreover, selection of free control parameters in order to improve effectiveness of the control, by emphasizing the action of the most controllable and observable modes, is discussed. To achieve such an objective, the control design procedure, still based on an $\mathscr{H}_{2}$ approach, adopts an LMI (linear matrix inequality) technique to compute the control parameters to allow the user to select the design weights based on various design requirements or by taking into account modal controllability/observability indices. The paper reports experimental results of broadband vibration reduction for a stiffened aeronautical panel controlled with piezoelectric actuators.

A final consideration is in order in this section. It is clear that the placement of the actuators and sensors is of paramount importance in the whole response of the flexible structure to external disturbances. Thus, an efficient control system should in general address also the problem of the optimal allocation of actuators and sensors, for example, based on maximizing vibrational energy reduction. However, in practical situations the position of sensors and actuators is often constrained by different considerations (geometry, weight limitations, safety, and cost); thus the control designer has very limited degrees of freedom in deciding sensors and actuators position and even number. For this reason, in this paper the location of sensors and actuators is assumed to be given.

\section{The Model}

In this paper a flexible structure with $m$ control inputs and $m+n_{d}$ measured (control) outputs is considered. The structure is assumed to be infinite-dimensional, linear, and time invariant. Since in general $n_{d} \geq 0$, the system is not necessarily square, that is, it may have more outputs than inputs. This assumption is quite reasonable in flexible systems control applications, where actuators are heavy and expensive, while sensors (accelerometers) are light, small, and relatively cheap.

As extensively discussed in [5], the mathematical model of a flexible structure can be written as

$$
\begin{gathered}
\dot{x}=A x+\bar{B}_{u} \bar{u}, \\
\bar{y}=\bar{C}_{y} x,
\end{gathered}
$$

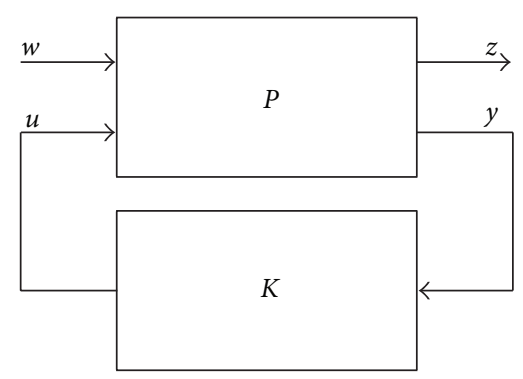

FIGURE 1: Standard control problem scheme.

where $x=\left(x_{1}^{T} x_{2}^{T}\right)^{T} \in \mathbb{R}^{2 n}$ is the state vector, $\bar{u} \in \mathbb{R}^{m}$ the control input, and $\bar{y} \in \mathbb{R}^{m+n_{d}}$ the measured (control) output. Obviously, since we are approximating an infiniteorder system, the number of retained modes $n$ is generally very high (with systems of order of hundreds). By resorting to the "modal coordinates" the matrices of the state space model assume the form

$$
\begin{gathered}
A=\left(\begin{array}{cc}
0 & I \\
-\Omega & -\Lambda
\end{array}\right), \\
\bar{B}_{u}=\left(\begin{array}{c}
0 \\
\bar{B}_{u_{2}}
\end{array}\right), \\
\bar{C}_{y}=\left(\begin{array}{lll}
0 & \bar{B}_{d_{2}}^{T} & \bar{B}_{u_{2}}^{T}
\end{array}\right),
\end{gathered}
$$

where

$$
\Omega=\operatorname{diag}\left(\omega_{1}^{2}, \ldots, \omega_{n}^{2}\right), \quad \Lambda=\operatorname{diag}\left(2 \zeta_{1} \omega_{1}, \ldots, 2 \zeta_{n} \omega_{n}\right),
$$

with $\omega_{i}$ and $\zeta_{i}>0$ being the natural frequency and damping coefficient of the $i$ th mode $(i=1, \ldots, n)$. In this case $x_{1}$ are the modal displacements and $x_{2}$ the modal velocities. Moreover, assuming that the velocity measurement is available, the output matrix $\bar{C}_{y}$ has the form (4). By following the gray-box approach proposed by Cavallo et al. [5], the model above can be accurately identified through a measurement campaign carried out on the structure. The gray-box approach assumes that the structure of the system to identify is exactly the one in (2)-(4), and this assumption is of paramount importance in the design of the controller, as it will be illustrated in Section 3.

\section{The Control Strategy}

In order to design a stabilizing controller for the flexible system it is convenient to resort to the standard TITO (twoinput-two-output) control problem framework depicted in Figure 1. In this figure, $P$ is the system to control, with measured output $y$, control input $u$, generalized disturbance $w$, and performance output $z$. Augmenting the flexible system with suitable fictitious "disturbance" and "performance" 
matrices, the following generalized plant $P$ is obtained:

$$
\begin{gathered}
\dot{x}=A x+B_{w} w+B_{u} u, \\
z=C_{z} x+D_{12} u, \\
y=C_{y} x+D_{21} w
\end{gathered}
$$

where $w \in \mathbb{R}^{m+n_{d}+n}$ is a fictitious disturbance, $z \in \mathbb{R}^{m+n}$ a fictitious performance output, and

$$
\begin{gathered}
u=R_{1}^{1 / 2} \bar{u} \in \mathbb{R}^{m}, \quad y=R_{2}^{-1 / 2} \bar{y} \in \mathbb{R}^{m+n_{d}}, \\
B_{u}=\bar{B}_{u} R_{1}^{-1 / 2}=\left(\begin{array}{c}
0 \\
B_{u_{2}}
\end{array}\right), \\
C_{y}=R_{2}^{-1 / 2} \bar{C}_{y}=\left(\begin{array}{lll}
0 & B_{d_{2}}^{T} & B_{u_{2}}^{T}
\end{array}\right),
\end{gathered}
$$

with $R_{1}$ and $R_{2}$ being the weighting matrices to be selected. The matrices $B_{w}$ and $C_{z}$ are $2 n \times\left(m+n_{d}+n\right)$ and $(m+n) \times 2 n$ real matrices, respectively, with the following structure:

$$
\begin{gathered}
B_{w}=\left(\begin{array}{ll}
0_{n \times n} & 0_{n \times\left(n_{d}+m\right)} \\
W^{1 / 2} & 0_{n \times\left(n_{d}+m\right)}
\end{array}\right), \\
C_{z}=\left(\begin{array}{cc}
0_{n \times n} & Q^{1 / 2} \\
0_{m \times n} & 0_{m \times n}
\end{array}\right),
\end{gathered}
$$

where the matrices $W$ and $Q$ are to be selected. Finally, for the sake of simplicity, the matrices $D_{12}$ and $D_{21}$ are chosen as to satisfy the following conditions:

$$
D_{12}^{T} C_{z}=0, \quad B_{w} D_{21}^{T}=0 .
$$

Although not essential, conditions (13) are standard in $\mathscr{H}_{2}$ or $\mathscr{H}_{\infty}$ control [6]. It is possible to remove them by slightly increasing the complexity of the control strategy. A possible selection is

$$
D_{12}=\left(\begin{array}{c}
0_{n \times m} \\
D_{12_{2}}
\end{array}\right), \quad D_{21}=\left(\begin{array}{ccc}
0_{n_{d} \times n} & D_{21_{1}} & 0_{n_{d} \times m} \\
0_{m \times n} & 0_{m \times n_{d}} & D_{21_{2}}
\end{array}\right),
$$

where

$$
\begin{gathered}
R_{1}=D_{12}^{T} D_{12}=D_{12_{2}}^{T} D_{12_{2}}>0, \\
R_{2}=\left(\begin{array}{cc}
D_{21_{1}} D_{21_{1}}^{T} & 0_{n_{d} \times m} \\
0_{m \times n_{d}} & D_{21_{2}} D_{21_{2}}^{T}
\end{array}\right)=\left(\begin{array}{cc}
R_{2_{1}} & 0 \\
0 & R_{2_{2}}
\end{array}\right)>0 .
\end{gathered}
$$

Finally, let $T_{z w}$ denote the lower LFT (linear fractional transformation) $T_{z w}=\mathcal{F}_{l}(P, K)[6]$.

Before computing the controller, a discussion on the required properties of the controller is in order. As stated in the introduction, stable bandpass controllers are preferred options in the control of flexible systems, due to their ability to filter out accelerometer biases. The following lemma is useful for characterizing the state space representation of a general class of bandpass systems.

Lemma 1. Let $H(s)$ be a linear time invariant (LTI) $2 n$ (McMillan) degree system with minimal state space representation

$$
H(s)=\left[\begin{array}{l|l}
A & B \\
\hline C & 0
\end{array}\right]
$$

where

$$
A=\left(\begin{array}{cc}
0 & I \\
A_{1} & A_{2}
\end{array}\right), \quad B=\left(\begin{array}{c}
0 \\
B_{2}
\end{array}\right), \quad C=\left(\begin{array}{ll}
0 & C_{2}
\end{array}\right),
$$

where all the matrices are partitioned assuming the state to be split into two $n$-dimensional substates and the square $n \times n$ matrix $A_{1}$ is invertible. Then, the system $H(s)$ is bandpass, in the sense that

$$
\lim _{s \rightarrow 0} H(s)=\lim _{s \rightarrow \infty} H(s)=0 .
$$

Proof. Although the lemma is an extension of Theorem 1 in Cavallo et al. [3], it can be easily proved observing that

$$
\lim _{s \rightarrow 0}(s I-A)^{-1}=\left(\begin{array}{cc}
A_{1}^{-1} A_{2} & A_{1}^{-1} \\
-I & 0
\end{array}\right) .
$$

Thus, direct computation shows that the static gain is zero. The high-frequency behavior is trivial.

The above lemma has a key role in our context. Indeed, it guarantees that a controller with a bandpass frequency shape, as required in the introduction, can be imposed by satisfying the rather mild conditions (18). Thus, the lemma is useful to generate different families of bandpass controllers.

The last issue to address in order to design a useful controller for flexible structures is stability of the controller itself. It is well known that inserting unstable elements into the control loop increases sensitivity to disturbances and calls for high-bandwidth controller, thus also increasing the effect of measurement noise [7]. Moreover, in the case of flexible structures the high-bandwidth requirement conflicts with the need to avoid spillover effects.

A first possible family of controllers is obtained in the case of square plants, that is, when $n_{d}=0$. Indeed, in this case it is possible to compute the explicit solution of a stable stabilizing controller satisfying an $\mathscr{H}_{\infty}$ index. Preliminarily, the following lemma is needed.

Lemma 2. Consider the system (6)-(8), assume, $n_{d}=0$, and let $X_{\infty}$ and $Y_{\infty}$ be $(2 n \times 2 n)$ positive semidefinite matrices satisfying

$$
\begin{aligned}
& A^{T} X_{\infty}+X_{\infty} A+X_{\infty}\left(\gamma^{-2} B_{w} B_{w}^{T}-B_{u} B_{u}^{T}\right) X_{\infty}+C_{z}^{T} C_{z}=0, \\
& A Y_{\infty}+Y_{\infty} A^{T}+Y_{\infty}\left(\gamma^{-2} C_{z}^{T} C_{z}-C_{y}^{T} C_{y}\right) Y_{\infty}+B_{w} B_{w}^{T}=0,
\end{aligned}
$$

for a given $\gamma>0$. Then, a necessary condition for the solution of the AREs (21) is to be of the form

$$
X_{\infty}=\beta^{2} Y_{\infty}^{-1},
$$

where $\beta<\gamma$ is a real scalar, that is, $X_{\infty}$ and $Y_{\infty}$ are block diagonal.

Although rather technical, Lemma 2 is useful to characterize the closed-form solution to an $\mathscr{H}_{\infty}$ control problem, as stated by the following theorem. 
Theorem 3. Consider the system (6)-(8), with matrices given by (2), (10), and (11), assume, $n_{d}=0$, let the pair $\left(A, B_{u}\right)$ be stabilizable and the pair $\left(A, C_{y}\right)$ detectable, and let

$$
B_{w}=\left(\begin{array}{cc}
0 & 0 \\
B_{w_{2}} & 0
\end{array}\right), \quad C_{y}=\left(\begin{array}{cc}
0 & B_{w_{2}}^{T} \\
0 & 0
\end{array}\right),
$$

where

$$
B_{w_{2}}=\left(\alpha \Lambda+B_{u_{2}} B_{u_{2}}^{T}\right)^{1 / 2}
$$

and $\alpha>0$ is a scalar parameter.

Then, the following controller is strongly stabilizing and has $m$ zeros at the origin

$$
K_{\infty}(s, \gamma)=\left[\begin{array}{c|c}
A_{\infty} & B_{u} \\
\hline-\frac{\gamma^{2}}{\gamma^{2}-2} B_{u}^{T} & 0
\end{array}\right],
$$

where

$$
A_{\infty}=\left(\begin{array}{cc}
0 & I \\
-\Omega & -\Lambda_{\infty}
\end{array}\right)
$$

and

$$
\Lambda_{\infty}=\left(1-\frac{2}{\gamma^{2}}\right) \Lambda+2 \frac{\gamma^{2}-1}{\gamma^{2}-2} \sqrt{\frac{\gamma^{2}-1}{\gamma^{2}}} B_{u_{2}} B_{u_{2}}^{T} .
$$

Moreover, if

$$
\alpha=2 \sqrt{1-\gamma^{-2}}
$$

the LFT $T_{z w}$ satisfies

$$
\left\|T_{z w}\right\|_{\infty}<\gamma .
$$

Finally, inf $\gamma=\sqrt{2}$ and the controller

$$
K_{\mathrm{opt}}=B_{u_{2}}^{T}\left(B_{u_{2}} B_{u_{2}}^{T}\right)^{\dagger} B_{u_{2}},
$$

where $(\cdot)^{\dagger}$ denotes the pseudoinverse, achieves the optimal value

$$
\min _{K \in \mathcal{K}}\left\|T_{z w}\right\|_{\infty}=\sqrt{2},
$$

where $\mathcal{K}$ is the set of stable, stabilizing controllers with $m$ zeros at the origin $s=0$.

The proofs of Lemma 2 and Theorem 3 can be found in Cavallo et al. [5]. They are based on the classic solution to the $\mathscr{H}_{\infty}$ problem involving two coupled Riccati equations [6], while the lower bound for $\gamma$ is obtained by resorting to the theory of singularly perturbed systems. It is worth noticing that the above theorem yields as a byproduct the closed-form solution of the $\mathscr{H}_{2}$ problem, obtained by letting $\gamma \rightarrow \infty$.

Corollary 4. With the same hypotheses as those in Theorem 3, the controller

$$
K_{2}(s)=\left[\begin{array}{c|c}
A-2 B_{u} B_{u}^{T} & B_{u} \\
\hline-B_{u}^{T} & 0
\end{array}\right]
$$

minimizes the norm $\left\|T_{z w}\right\|_{2}$.
Proof. It is sufficient to let $\gamma \rightarrow \infty$ in the controller in Theorem 3.

Finally, by resorting to the equations for the parameterization of all the stabilizing controllers [8], it is possible to give a general expression for the whole family of $\mathscr{H}_{\infty}$ optimal controllers and $\mathscr{H}_{2}$ suboptimal controllers as follows.

Let

$$
Q(s)=C_{q}\left(s I-A_{q}\right)^{-1} B_{q}+D_{q}
$$

be a stable transfer matrix with $\|Q\|_{\infty}<\gamma$. Then, the family of controllers such that $\left\|T_{z w}\right\|_{\infty}<\gamma$ is

$$
K_{\infty, Q}(s)=\left[\begin{array}{c|c}
A_{\infty, Q} & B_{\infty, Q} \\
\hline C_{\infty, Q} & D_{q}
\end{array}\right],
$$

where

$$
\begin{aligned}
& A_{\infty, Q}=\left(\begin{array}{cc}
A_{\infty}-\frac{\gamma^{2}-1}{\gamma^{2}-2} B_{u} D_{q} B_{u}^{T} & \frac{\gamma^{2}-1}{\gamma^{2}-2} B_{u} C_{q} \\
-B_{q} B_{u}^{T} & A_{q}
\end{array}\right), \\
& B_{\infty, Q} \quad=\left(\begin{array}{cc}
\left.\frac{\gamma^{2}-1}{\gamma^{2}-2} B_{u}\left(D_{q}+\frac{\gamma}{\sqrt{\gamma^{2}-1}} I\right)\right), \\
B_{q}
\end{array}\right) \\
& C_{\infty, Q} \quad=\left(-\left(D_{q}+\frac{\gamma}{\sqrt{\gamma^{2}-1}} I\right) B_{u}^{T} B_{q}\right) .
\end{aligned}
$$

Analogously, an (simpler) expression for the $\mathscr{H}_{2}$ suboptimal controller may be obtained as follows:

$$
K_{2, Q}(s)=\left[\begin{array}{cc|c}
A-2 B_{u} B_{u}^{T} & B_{u} C_{q} & B_{u} \\
-B_{q} B_{u}^{T} & A_{q} & B_{q} \\
\hline-B_{u}^{T} & C_{q} & 0
\end{array}\right],
$$

where, it has been, obviously, assumed that $D_{q}=0$.

In the general case of $n_{d} \neq 0$ the above formulation does not hold any longer, and the design of the controller has to be redefined. The main reason is that the Riccati equations to be solved for the $\mathscr{H}_{\infty}$ problem are strongly coupled. The choice of a stabilizing controller with the structure in Lemma 1 can be carried out by exploiting the following theorem.

Theorem 5. Any system of the form (6)-(8) with matrices given by (2), (10), and (11) is closed-loop stabilized by a controller with state space representation

$$
K(s)=\left[\begin{array}{c|c}
A_{c} & B_{c} \\
\hline C_{c} & 0
\end{array}\right],
$$

where

$$
\begin{aligned}
& B_{c}=\left(\begin{array}{cc}
0_{m \times n} & 0_{m \times n} \\
Y_{2} B_{d_{2}} R_{2_{1}}^{-1} & Y_{2} B_{u_{2}} R_{2_{2}}^{-1}
\end{array}\right), \\
& C_{c}=-R_{1}^{-1}\left(\begin{array}{ll}
0_{m \times n} & B_{u_{2}}^{T} X_{2}
\end{array}\right), \\
& A_{c}=A+B_{u} C_{c}-B_{c} C_{y},
\end{aligned}
$$


where $X_{2}$ and $Y_{2}$ are $n \times n$ diagonal matrices with nonnegative entries.

Proof. Let

$$
\begin{gathered}
Q=2 X_{2} \Lambda+X_{2} B_{u_{2}} R_{1}^{-1} B_{u_{2}}^{T} X_{2}, \\
W=2 Y_{2} \Lambda+Y_{2}\left(B_{d_{2}} R_{2_{1}}^{-1} B_{d_{2}}^{T}+B_{u_{2}} R_{2_{2}}^{-1} B_{u_{2}}^{T}\right) Y_{2} .
\end{gathered}
$$

It is clear that both $Q$ and $W$ are positive semidefinite for any nonnegative matrices $X_{2}$ and $Y_{2}$. Moreover, by defining the matrices

$$
\begin{gathered}
X=\left(\begin{array}{cc}
\Omega X_{2} & 0 \\
0 & X_{2}
\end{array}\right), \\
Y=\left(\begin{array}{cc}
\Omega^{-1} Y_{2} & 0 \\
0 & Y_{2}
\end{array}\right),
\end{gathered}
$$

it is easy to show that $X=\operatorname{Ric}\left(H_{2}\right)$ and $Y=\operatorname{Ric}\left(J_{2}\right)$, where, for a given Hamiltonian matrix $Z$, $\operatorname{Ric}(Z)$ denotes the stabilizing solution of the Riccati equation associated to the Hamiltonian matrix [6], and the Hamiltonian matrices $\mathrm{H}_{2}$ and $J_{2}$ are

$$
\begin{aligned}
& H_{2}=\left(\begin{array}{cc}
A & -B_{u} R_{1}^{-1} B_{u}^{T} \\
-\widetilde{Q} & -A^{T}
\end{array}\right), \\
& J_{2}=\left(\begin{array}{cc}
A^{T} & -C_{y}^{T} R_{2}^{-1} C_{y} \\
-\widetilde{W} & -A
\end{array}\right) .
\end{aligned}
$$

Finally, the matrices $\widetilde{Q}$ and $\widetilde{W}$ are

$$
\begin{aligned}
\widetilde{Q} & =\left(\begin{array}{ll}
0 & 0 \\
0 & Q
\end{array}\right), \\
\widetilde{W} & =\left(\begin{array}{cc}
0 & 0 \\
0 & W
\end{array}\right) .
\end{aligned}
$$

Thus, for any matrices $X_{2}$ and $Y_{2}$ the controller (38) solves an $\mathscr{H}_{2}$ problem and is therefore stabilizing.

Controller stability is not guaranteed by the above theorem; thus it must be enforced as follows. Preliminarily, define

$$
\begin{aligned}
\widetilde{B}_{X} & =B_{u} R_{1}^{-1} B_{u}^{T}, \\
\widetilde{B}_{Y} & =C_{y} R_{2}^{-1} C_{y}^{T} .
\end{aligned}
$$

From the above formulation it is easy to deduce the following theorem.

Theorem 6. Consider the system $K_{s}(s)$ with minimal state space representation

$$
K_{s}(s)=\left[\begin{array}{c|c}
A_{c} & B_{c} \\
\hline C_{c} & 0
\end{array}\right],
$$

where the matrices $A_{c}, B_{c}$, and $C_{c}$ are given in (38). If the diagonal matrices $X_{2}$ and $Y_{2}$ are chosen so as to satisfy the LMI

$$
\begin{gathered}
X_{2} \widetilde{B}_{X}+\widetilde{B}_{X} X_{2}+Y_{2} \widetilde{B}_{Y}+\widetilde{B}_{Y} Y_{2}>0 \\
X_{2}>0 \\
Y_{2}>0
\end{gathered}
$$

then the system $K_{s}(s)$ strongly stabilizes the system (6)-(8) and is bandpass.

Proof. The theorem can be easily proved by considering the Lyapunov function

$$
V(x)=x^{T}\left(\begin{array}{cc}
\Omega^{-1} & 0 \\
0 & I
\end{array}\right) x .
$$

The rest of the theorem follows from Theorem 5 and Lemma 1.

The above theorem shows that a parametrization of stable, stabilizing, and bandpass controller is obtained, with $2 n$ free parameters, namely, the diagonal entries of the matrices $X_{2}$ and $Y_{2}$. Since these matrices define the controller gain, a further constraint in the form of LMI

$$
\operatorname{tr}\left(X_{2}\right)+\operatorname{tr}\left(Y_{2}\right)<\delta
$$

where $\operatorname{tr}(X)$ is the trace of the matrix $X$ and $\delta$ is an upper bound on the controller gain depending on the problem, can be imposed. Moreover, also the matrices $R_{1}$ and $R_{2}$ have still to be selected. Expressions (39) and (40) can suggest how to choose the degrees of freedom. According to them, $X_{2}$ and $Y_{2}$ can weigh modes on which to operate, while the matrices $R_{1}$ and $R_{2}$ define the authority of the control.

The above considerations can be summarized in a procedure for controller design, namely,

(1) preliminarily, collect the diagonal elements of $X_{2}$ and $Y_{2}$ in the vector $\xi=\left(x_{2_{11}}, \ldots, x_{2_{n n}}, y_{2_{11}}, \ldots, y_{2_{n n}}\right)^{T}$, define a vector of performance weights $c \in \mathbb{R}^{n}$, whose $j$ th element is the weight of the $j$ th mode,

(2) solve the optimization problem

$$
\max \quad\left(c^{T} c^{T}\right) \xi
$$

subject to

$$
\begin{aligned}
& X_{2}(\xi) \widetilde{B}_{X}+\widetilde{B}_{X} X_{2}(\xi)+Y_{2}(\xi) \widetilde{B}_{Y} \\
& \quad+\widetilde{B}_{Y} Y_{2}(\xi)+2 \Lambda>0, \\
& X_{2}(\xi)>0, \\
& Y_{2}(\xi)>0, \\
& 1_{n}^{T} X_{2}(\xi) 1_{n}+1_{n}^{T} Y_{2}(\xi) 1_{n}<\delta,
\end{aligned}
$$

where $\delta>0$ and $1_{n}$ is the $n$-dimensional column vector with all elements equal to 1 ;

(3) compute the controller according to (37)-(38).

In the above procedure, the scalar $\delta>0$ is chosen to limit the controller gain since (54) can be written as

$$
\operatorname{tr}\left(X_{2}\right)+\operatorname{tr}\left(Y_{2}\right)<\delta
$$

Obviously, in the above algorithm $c_{j}$, the $j$ th element of $c$ can assume any real value. For instance, choosing $c_{1}=10$, 
$c_{2}=1$, and $c_{3}=0.1$ would pose different penalties on different modes, thus focussing the control action more on mode one than on mode two and on mode three. Cavallo et al. [9] showed that the optimal choice of the input scaling matrices is performed based on the controllability Gramian of the open-loop and closed-loop systems. However, it is clear that the efficiency of the control action depends on both the controllability and the observability Gramians. As an example, a well-known strategy for model order reduction [10] consists of the computation of a balanced realization and a reduction based on neglecting the modes associated with the Hankel singular values, that is, square roots of the eigenvalues of the product of the controllability Gramian and the observability Gramian, with the lowest magnitude. This kind of consideration leads to the idea of selecting the modal performance weights in (50) based on the Hankel singular values. Another aspect of the presented control strategy to highlight is the selection of the matrices $R_{1}$ and, even more importantly, $R_{2}$ in (15) and (16), respectively. The former is responsible for the authority of the control. The latter is responsible for the relative weights of colocated measurements with respect to noncolocated ones. By varying the magnitude of $R_{2_{1}}$ relative to $R_{2_{2}}$ the case of noncolocated feedback can be considered with stability guaranteed.

\section{Experimental Results}

Control procedures described in the previous section have been experimentally tested on a very complex flexible structure. In particular, the selected test article is a fuselage skin panel of a BOEING 717 (depicted in Figure 2). The panel is stiffened by two bulkheads and three orthogonal stiffeners riveted to the rear of the panel itself. In order to reproduce the conditions usually adopted in the structural testing phase, the panel has been suspended by a couple of soft springs to simulate free boundary conditions. In the experiments presented in this section piezoelectric ceramic actuators have been used both for producing the primary disturbance field and for generating the control forces on the structure aimed at counteracting the effects of the primary field.

4.1. Square Case. In detail, the control inputs are produced using three of the four piezoelectric patches bonded on the panel surface. The output acceleration is measured by means of the three colocated accelerometers placed on the rear part of the panel behind the piezoelectric actuators. The structure has a high modal density in the considered frequency range $\Omega=[100,600] \mathrm{Hz}$ and a model with 148 modes has been identified as proposed by Cavallo et al. [11]. The experiments were performed by using a dSPACE rapid prototyping realtime control system with 16-bit A/D channels and 14-bit D/A channels at a sampling frequency of $20 \mathrm{kHz}$.

A fourth piezo is used only as a disturbance input so as to introduce a disturbance contribution not belonging to the range of the input matrix $B_{u}$ in (6).

The effectiveness of the control strategy has been evaluated comparing the frequency response functions (FRFs) from disturbance input to acceleration outputs and in terms

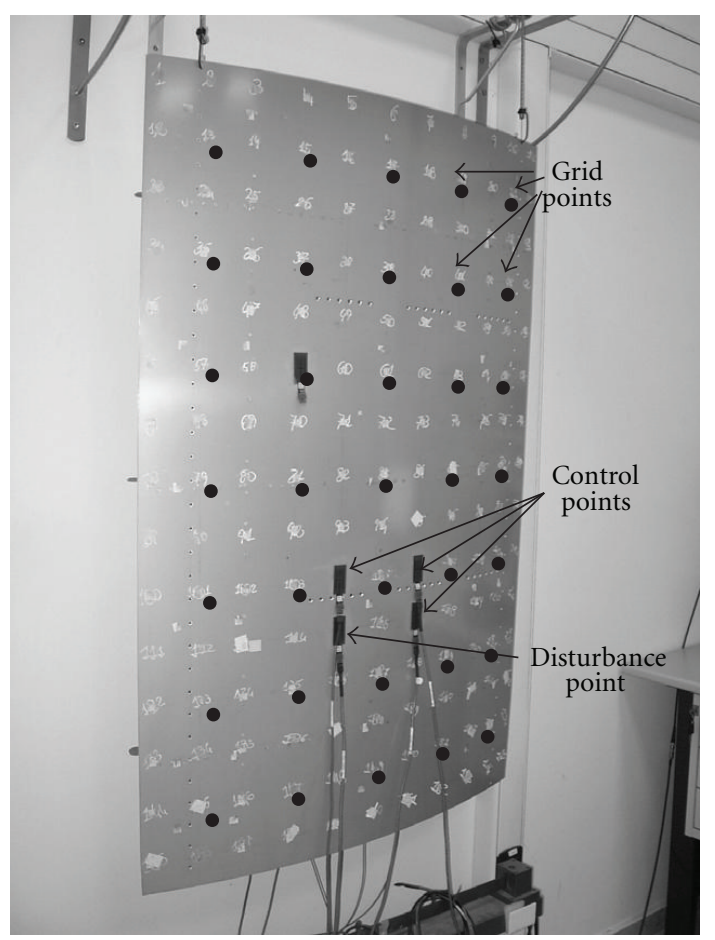

FIGURE 2: Front view of the panel with piezoelectric actuators and grid performance point.

of an energy-based index defined as follows. First, the energy of the measured acceleration in the given frequency range $\Omega$ has been estimated for the $k$ th of the five used sensors as

$$
J_{k}=\sqrt{\sum_{h=1}^{M}\left|\Phi_{k}\left(\omega_{h}\right)\right|^{2}}, \quad k=1, \ldots, 35,
$$

where $\Phi_{k}\left(\omega_{h}\right)$ is the acceleration spectrum measured at the $k$ th point at the frequency $\omega_{h} \in\left\{\omega_{1}, \ldots, \omega_{M}\right\}$, a set of $M=$ 2500 linearly spaced frequencies within $\Omega$. Then, the relative energy reduction at the $k$ th point has been quantified as

$$
R_{k \%}=\frac{J_{k, \mathrm{ol}}-J_{k, \mathrm{cl}}}{J_{k, \mathrm{ol}}} 100,
$$

where the subscripts "ol" and "cl" stand for "open loop" and "closed loop," respectively.

In a first experimental campaign, the optimal $\mathscr{H}_{\infty}$ and $\mathscr{H}_{2}$ controllers are tested. One of the known drawbacks in the application of optimal feedback control to vibration reduction problems is that the control action may focus only on the performance objectives, reducing vibrations only at the controlled points, while increasing vibration levels at other points of the structure. In order to check that the controller actually has a beneficial effect on the whole structure a matrix of 35 points evenly spaced on the panel has been considered, as shown in Figure 2.

A band-limited white noise in the range $[100,600] \mathrm{Hz}$ has been chosen as primary field, to excite all the system 


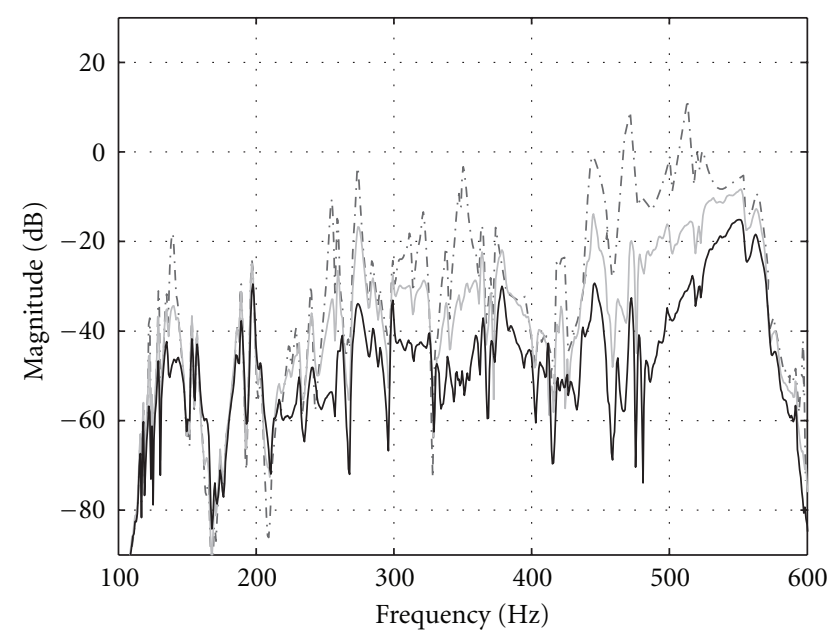

Figure 3: Acceleration in a colocated point of the panel: open loop (dashed grey line), $\mathscr{H}_{2}$ (solid grey line), and $\mathscr{H}_{\infty}$ (solid black line).

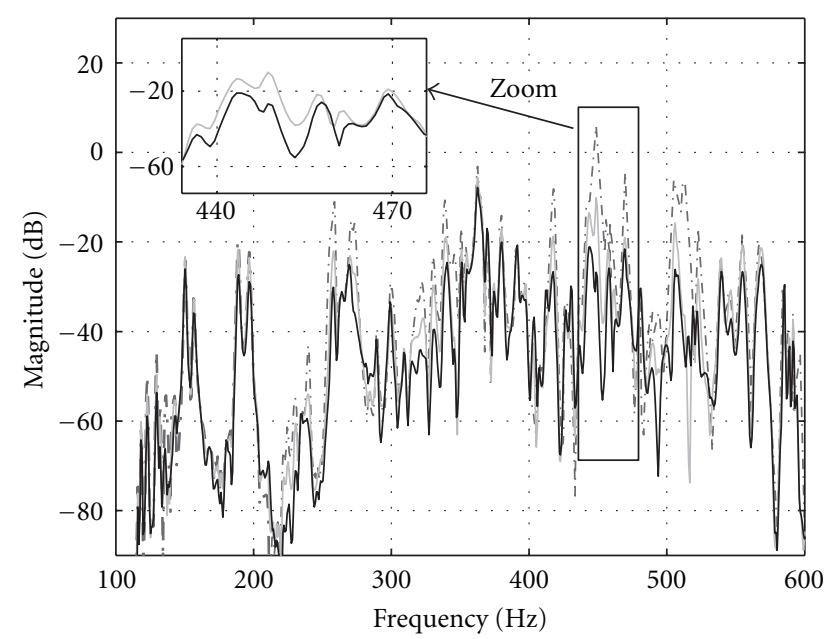

FIGURE 4: Acceleration in a noncolocated point of the panel: open loop (dashed grey line), $\mathscr{H}_{2}$ (solid grey line), and $\mathscr{H}_{\infty}$ (solid black line).

natural modes in the frequency range $\Omega$. The noise has been applied to the structure on all four input channels so that the disturbance has components both in the range of $B_{u}$ and off range. Figures 3 and 4 present results for both $\mathscr{H}_{2}$ and $\mathscr{H}_{\infty}$ cases. The $\mathcal{H}_{\infty}$ controller has been designed fixing $\gamma=1.5$ in (25). The figures show the acceleration spectrum measured in a control point and in a performance test point. Obviously, performance is better at the control points than at the test points, where a vibration reduction is still apparent, anyway.

Figures 5 and 6 report the values of the relative energy reduction index as defined in expression (57) at each grid point for the $\mathscr{H}_{2}$ and $\mathscr{H}_{\infty}$ cases, respectively, showing the effectiveness of both controllers on the whole panel. The average vibration reductions are $37.8 \%$ with the $\mathscr{H}_{2}$ controller $\mathscr{H}_{2}$ and $55.1 \%$ with the $\mathscr{H}_{\infty}$ controller almost uniformly on the whole panel, despite the small number of

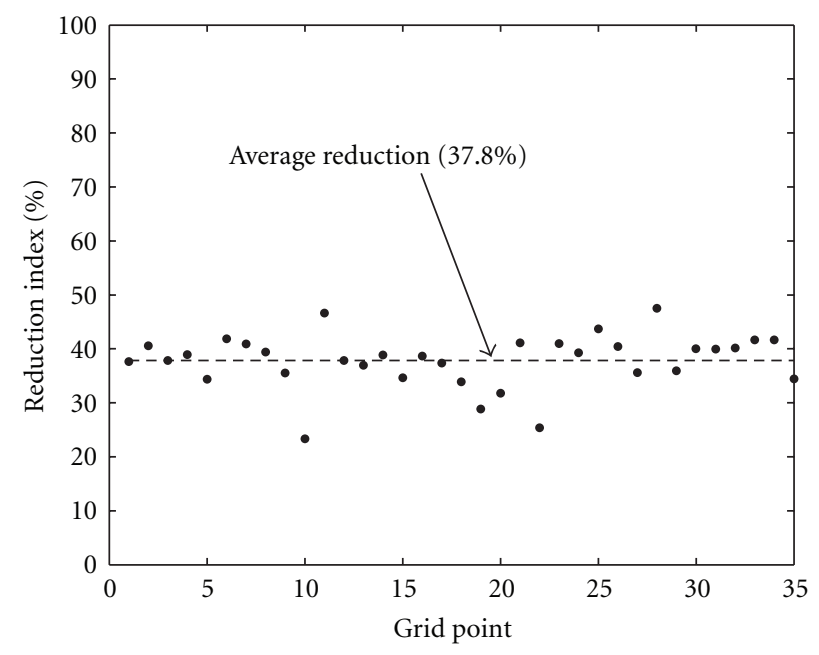

FIGURE 5: Reduction energy index at grid points of the panel with $\mathscr{H}_{2}$ controller.

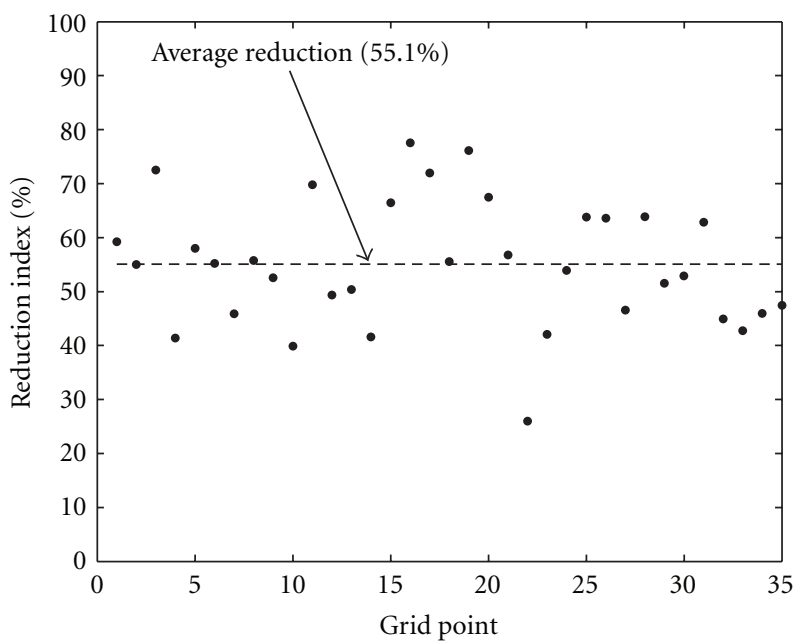

FIGURE 6: Reduction energy index at grid points of the panel with $\mathcal{H}_{\infty}$ controller.

control actuators, the high modal density, and the broadband nature of the disturbance.

Figures 7 and 8 further clarify the effectiveness of the proposed controllers reporting the values of the $J_{k}$ energy index in all the grid points with the same color scale in order to compare open loop with closed loop.

Finally, Figure 9 presents the time history of a control input (piezoelectric actuator voltage) in both the $\mathscr{H}_{2}$ and the $\mathscr{H}_{\infty}$ cases, showing that the control voltage with the $\mathscr{H}_{\infty}$ controller is generally higher than the $\mathscr{H}_{2}$ case (this explains why the $\mathscr{H}_{\infty}$ controller behaves better). Nevertheless, in both cases the input voltage is far below the saturation limits of the actuators $(200 \mathrm{~V})$.

4.2. Rectangular Case. The next campaign of experiments addresses the case of more measurements than controls available. In this case, a fifth accelerometer is used to measure the acceleration in a performance point far from both control 

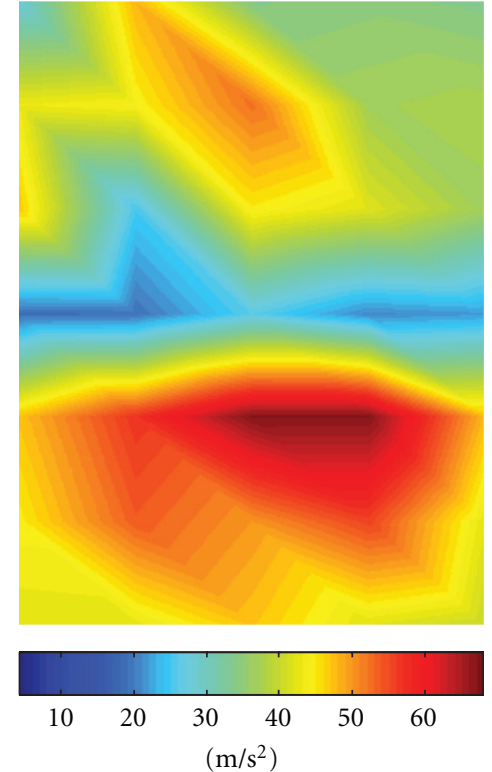

(a)

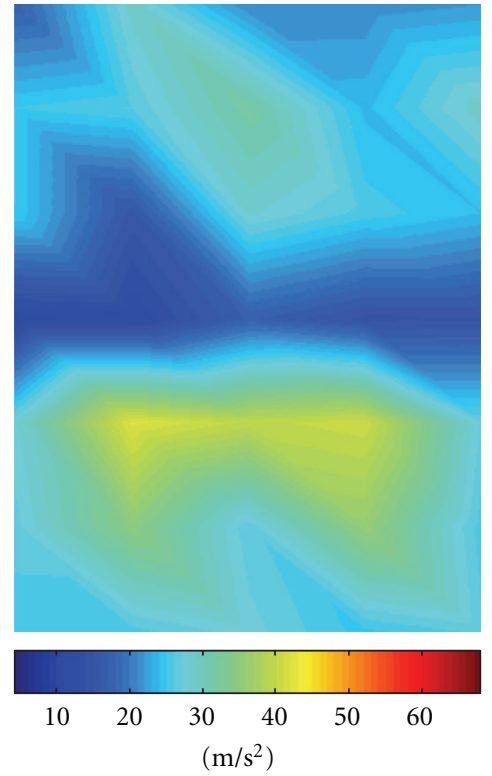

(b)

Figure 7: Performance of the $\mathscr{H}_{2}$ controller on the whole panel: open loop (a) and closed loop (b).

and disturbance inputs. Moreover, the noise has been applied to the structure only through the fourth piezo actuator not used as control input, thus the disturbance is totally out of the range of the input matrix $B_{u}$. Although this makes the two sets of experiments not comparable, this choice allows better highlighting of the feature of the controller when used with additional sensors located near the entry point of the primary field. Since the controller computation depends on the choice of modal performance weights and on the number of feedback sensors, the guidelines described at the end of Section 3 have been followed. As a consequence, three controllers have been computed and compared:
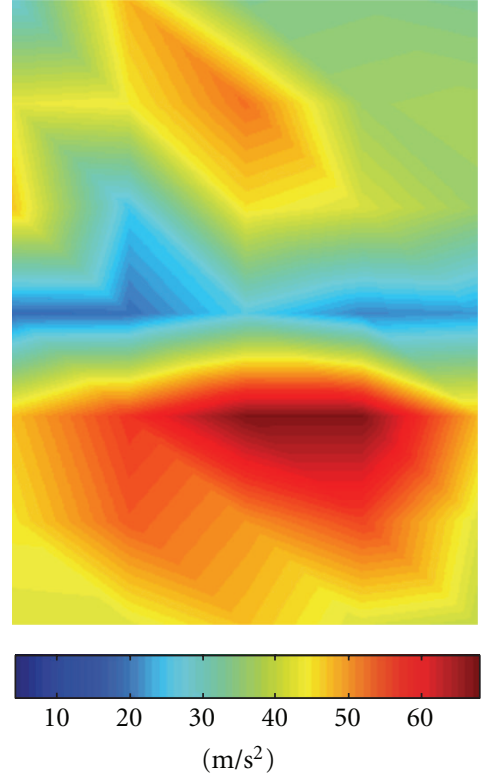

(a)
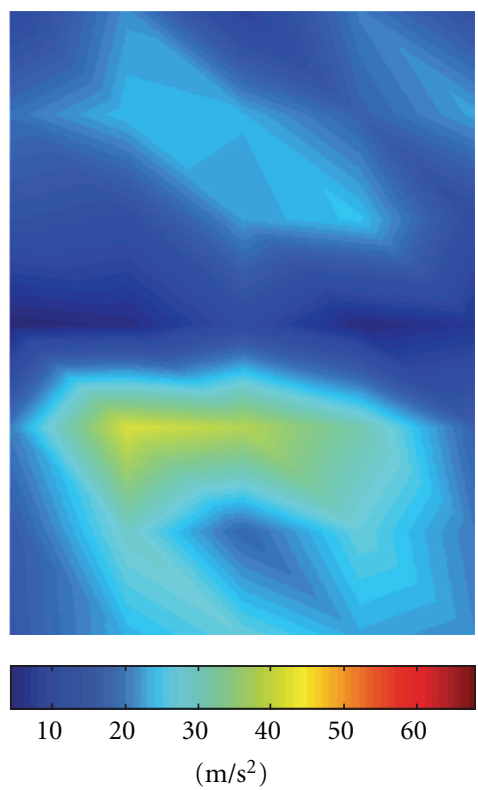

(b)

Figure 8: Performance of the $\mathscr{H}_{\infty}$ controller on the whole panel: open loop (a) and closed loop (b).

A: colocated controller with modal performance weights based on the Henkel singular values,

B: rectangular controller (with an additional sensor) with modal performance weights based on the Henkel singular values,

C: noncolocated controller with modal performance weights based on the Henkel singular values.

All these controllers use the same three piezoelectric actuators as control inputs and the four accelerometers as measured outputs and are designed by selecting the modal 


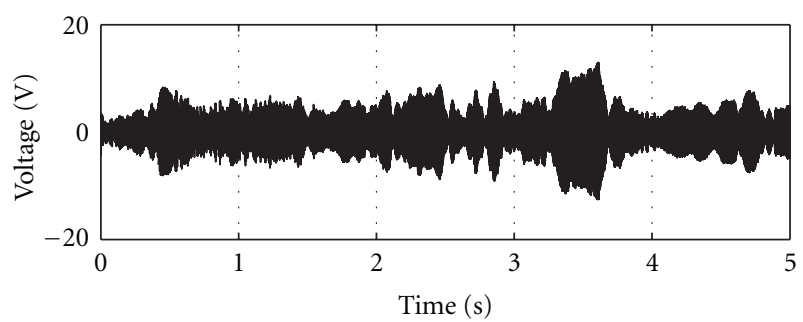

(a)

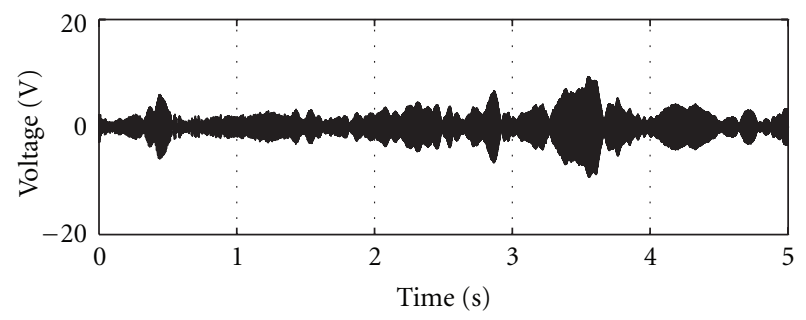

(b)

Figure 9: Control signals in the $\mathscr{H}_{\infty}$ case (a) and $\mathscr{H}_{2}$ case (b).

performance weights in (50) according to the Henkel singular values. However, controller A practically uses, as output accelerations, only the measurements provided by the three colocated sensors, since weighting matrices are selected as $R_{1}=I, R_{2_{1}}=10^{8}$, and $R_{2_{2}}=I$. The large value of $R_{2_{1}}$ makes the information of the fourth sensor meaningless for the control action. Controller B takes into account the problem of vibration reduction when information on the location of the application point of the disturbance force is available. Of course, in such a case, the optimal solution would be to locate an actuator/sensor pair in this entry point. However, assume that mounting the actuator in such a location is not possible for some reason, while placement of the sensor is still allowed. Under this assumption, controller $B$ uses the information provided by the fourth sensor by selecting the weighting matrices $R_{1}=I, R_{2_{1}}=1$, and $R_{2_{2}}=I$, where a lower value for $R_{2}$ has been set. The controller $C$ has been considered to assess the behaviour of the active vibration control system when the application at hand does not allow to place actuators and sensors in colocated pairs. In particular, it practically uses only the signal of the fourth sensor placed in the disturbance entry point, by selecting the weighting matrices $R_{1}=10^{-4} I, R_{2_{1}}=10^{-4}$, and $R_{2_{2}}=10^{8} \mathrm{I}$, where a large value for $R_{2_{2}}$ has been set. The rectangular noncolocated controller $\mathrm{C}$ has no information on what happens to the structure in the control points.

The performance obtained with the controllers above are evaluated both in terms of vibration reduction quantified according to the index in (57) and by comparing the FRFs from disturbance input point to a control point, the disturbance point, and the performance point. In the first experiment controller A, used as reference, has been compared to controller $B$. Table 1 shows that the reduction energy index for controller B is significantly higher in the disturbance and performance points. Moreover, the effects of controller B, compared with controller A and open loop, on
TABLe 1: Values of the reduction energy index $R_{k \%}$ in (57) obtained in the frequency range $[100,600] \mathrm{Hz}$ for disturbance and performance points.

\begin{tabular}{lcc}
\hline & Disturbance pt. & Performance pt. \\
\hline$R_{k \%}$ controller A & 30.74 & 30.58 \\
$R_{k \%}$ controller B & 39.16 & 39.50 \\
\hline
\end{tabular}

TABLE 2: Control energy reduction index $R_{e \%}$ for controllers B and C.

\begin{tabular}{lcc}
\hline & Controller B & Controller C \\
\hline Channel 1 & 11.18 & 56.35 \\
Channel 2 & 12.83 & 64.59 \\
Channel 3 & 10.14 & 75.57 \\
\hline
\end{tabular}

the entire frequency range $\Omega$ are shown in Figure 10, where over $20 \mathrm{~dB}$ of vibration reduction peaks are visible and no spillover appears.

The controllers have been also compared in terms of required control energy according to the index defined below. First, the energy $E_{s}$ of a generic discrete-time signal $s(k)$ has been evaluated as

$$
E_{s}=\sum_{k=1}^{N} s^{2}(k)
$$

where $N$ is a fixed number of samples equal for all experiments. Taking into account that the primary field has been randomly generated, the energy $E_{u, Y}^{r}$ of the $r$ th control signal in the experiment $Y$ ( $Y$ denotes the control label as listed above) has been normalized with respect to the actual energy of the disturbance $E_{d, Y}$ in the same experiment, that is,

$$
\widehat{E}_{u, Y}^{r}=\frac{E_{u, Y}^{r}}{E_{d, Y}}, \quad r=1,2,3, \quad Y=A, B, C .
$$

The control energy required by controllers $B$ and $C$ has been then compared to the one required by controller $\mathrm{A}$, by considering the control energy reduction index

$$
R_{e \%}=\frac{\widehat{E}_{u, A}^{r}-\widehat{E}_{u, X}^{r}}{\widehat{E}_{u, A}^{r}} 100, \quad r=1,2,3, \quad X=B, C .
$$

The results are summarized in Table 2, which shows that controller B requires less control energy than controller A despite its higher performance. It is apparent that the information from the additional sensor improves the control action.

In a subsequent experiment controller $\mathrm{C}$ has been tested. Although in the design phase the weighting matrices have been selected positive definite according to (15) and (16), in the real experiment, only the fourth sensor placed in the disturbance point has been physically used, in order to demonstrate the possibility to use the proposed controller as a true non-colocated one. The performance in terms of reduction energy index is reported in Table 3 . The reduction energy index values are lower than in the previous cases, but the same applies to the required control energy indicated 


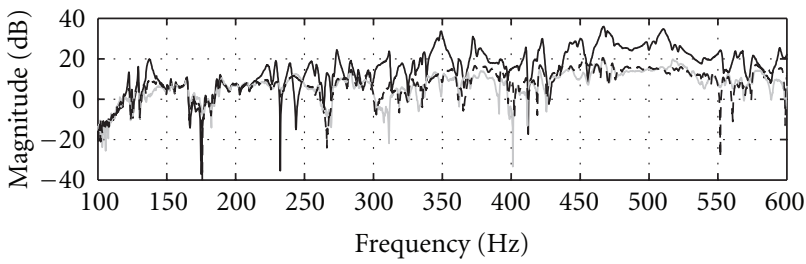

(a)

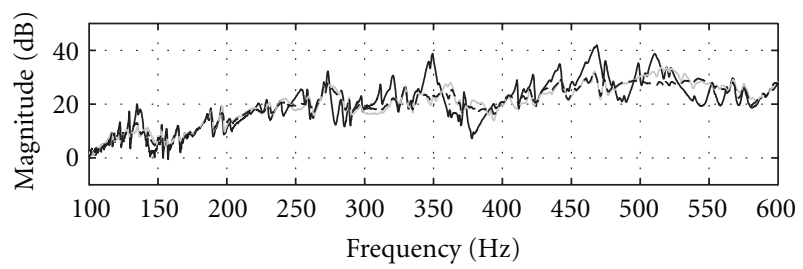

(b)

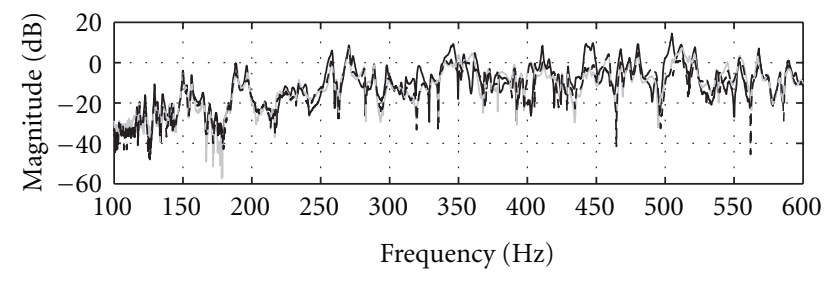

(c)

FIGURE 10: FRFs from disturbance input to acceleration output in a control point (a), in the disturbance point (b), and in the performance point (c): open loop (solid black lines), and controller A (solid grey lines), controller B (dashed black lines).

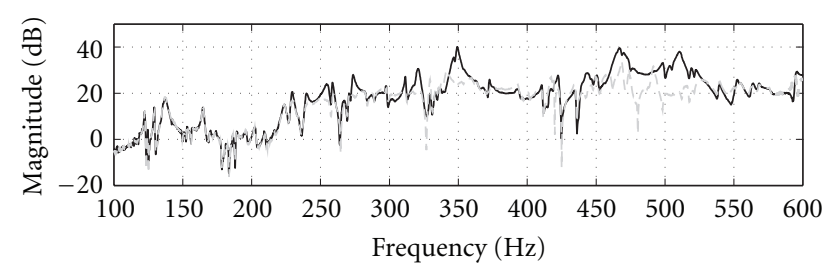

(a)

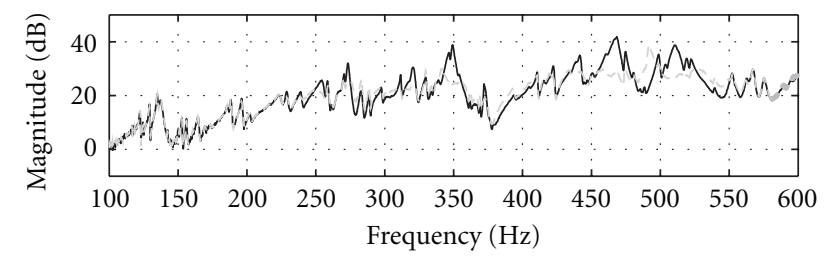

(b)

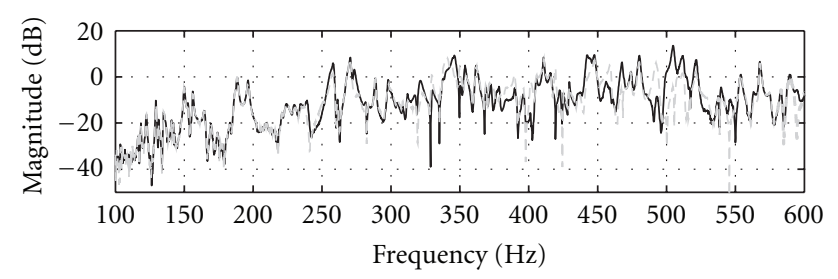

(c)

FIGURE 11: FRFs from disturbance input to acceleration output in a control point (a), in the disturbance point (b), and in the performance point (c): open loop (solid black lines) and noncolocated controller $\mathrm{C}$ (dashed grey lines).
TABLE 3: Values of the reduction energy index $R_{k \%}$ in (57) obtained for noncolocated controller in the frequency range $[100,600] \mathrm{Hz}$ for disturbance and performance points.

\begin{tabular}{lcc}
\hline & Disturbance pt. & Performance pt. \\
\hline$R_{k \%}$ controller C & 27.44 & 18.25 \\
\hline
\end{tabular}

in Table 2. Interestingly enough, even in this actually noncolocated control no spillover phenomenon occurs, as evident from the FRFs reported in Figure 11, still obtaining over $10 \mathrm{~dB}$ of vibration reduction peaks.

\section{Conclusions}

The paper reported a procedure for designing closed-form optimal $\left(\mathscr{H}_{\infty}\right.$ and $\left.\mathscr{H}_{2}\right)$ controllers for large-scale square systems, together with an extension to nonsquare plants to tackle the vibration reduction problem of flexible structure with the strong requirement of stable and bandpass controller. The original control law is here extended to the case of more sensors than actuators. The more complex design is tackled by solving an LMI problem, which allows also to select the performance weights based on controllability/observability indices. The experimental results obtained for a vibration reduction problem of a stiffened aeronautical panel controlled by piezoelectric actuators confirm the applicability of the strategy in actual systems and its advantages with respect to a controller assuming only colocated actuator/sensor pairs.

\section{References}

[1] M. Balas, "Trends in large space structure control theory: fodest hopes, widest dreams," IEEE Transactions on Automatic Control, vol. 27, pp. 522-535, 1982.

[2] A. Cavallo, G. De Maria, and R. Setola, "Sliding manifold approach for vibration reduction of flexible systems," Automatica, vol. 35, no. 10, pp. 1689-1696, 1999.

[3] A. Cavallo, G. De Maria, C. Natale, and S. Pirozzi, "Robust control of flexible structures with stable bandpass controllers," Automatica, vol. 44, no. 5, pp. 1251-1260, 2008.

[4] A. Cavallo, G. De Maria, C. Natale, and S. Pirozzi, " $\mathscr{H} \infty$ strongly stabilizing bandpass controllers for flexible systems," in Proceedings of the IEEE Conference on Decision and Control, pp. 6543-6548, 2006.

[5] A. Cavallo, G. De Maria, C. Natale, and S. Pirozzi, Active Control of Flexible Structures: From Modelling to Implementation, Springer, London, UK, 2010.

[6] K. Zhou and J.C. Doyle, Essentials of Robust Control, Prentice Hall, New Jersey, NJ, USA, 1995.

[7] J. Maciejowski, Multivariable Feedback Design, AddisonWesley, Wokingham, UK, 1989.

[8] D. C. Youla, J. J. Bongiorno, and C. N. Lu, "Singleloop feedback-stabilization of linear multivariable dynamical plants," Automatica, vol. 10, no. 2, pp. 159-173, 1974.

[9] A. Cavallo, G. De Maria, C. Natale, and S. Pirozzi, "On scaling matrices optimal selection for $\mathrm{H}_{2}$ strongly stabilizing bandpass controllers," in Proceedings of the IEEE Conference on Decision and Control, pp. 4749-4754, New Orleans, La, USA, 2007. 
[10] B. C. Moore, "Principal components analysis in linear systems: controllability, observability and model reduction," IEEE Transactions on Automatic Control, vol. AC-26, no. 1, pp. 1732, 1981.

[11] A. Cavallo, G. De Maria, C. Natale, and S. Pirozzi, "Graybox identification of continuous-time models of flexible structures," IEEE Transactions on Control Systems Technology, vol. 15, no. 5, pp. 967-981, 2007. 

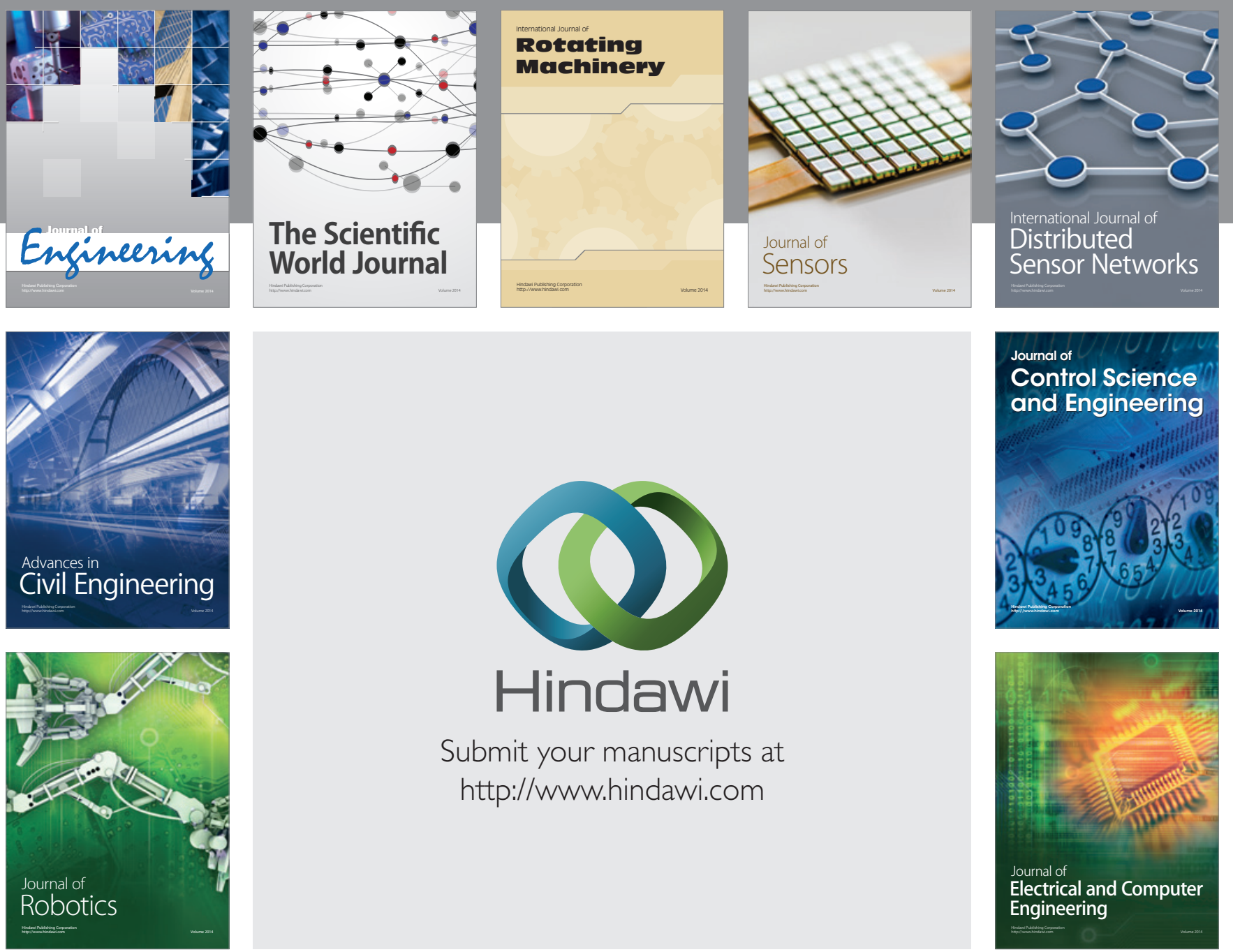

Submit your manuscripts at

http://www.hindawi.com
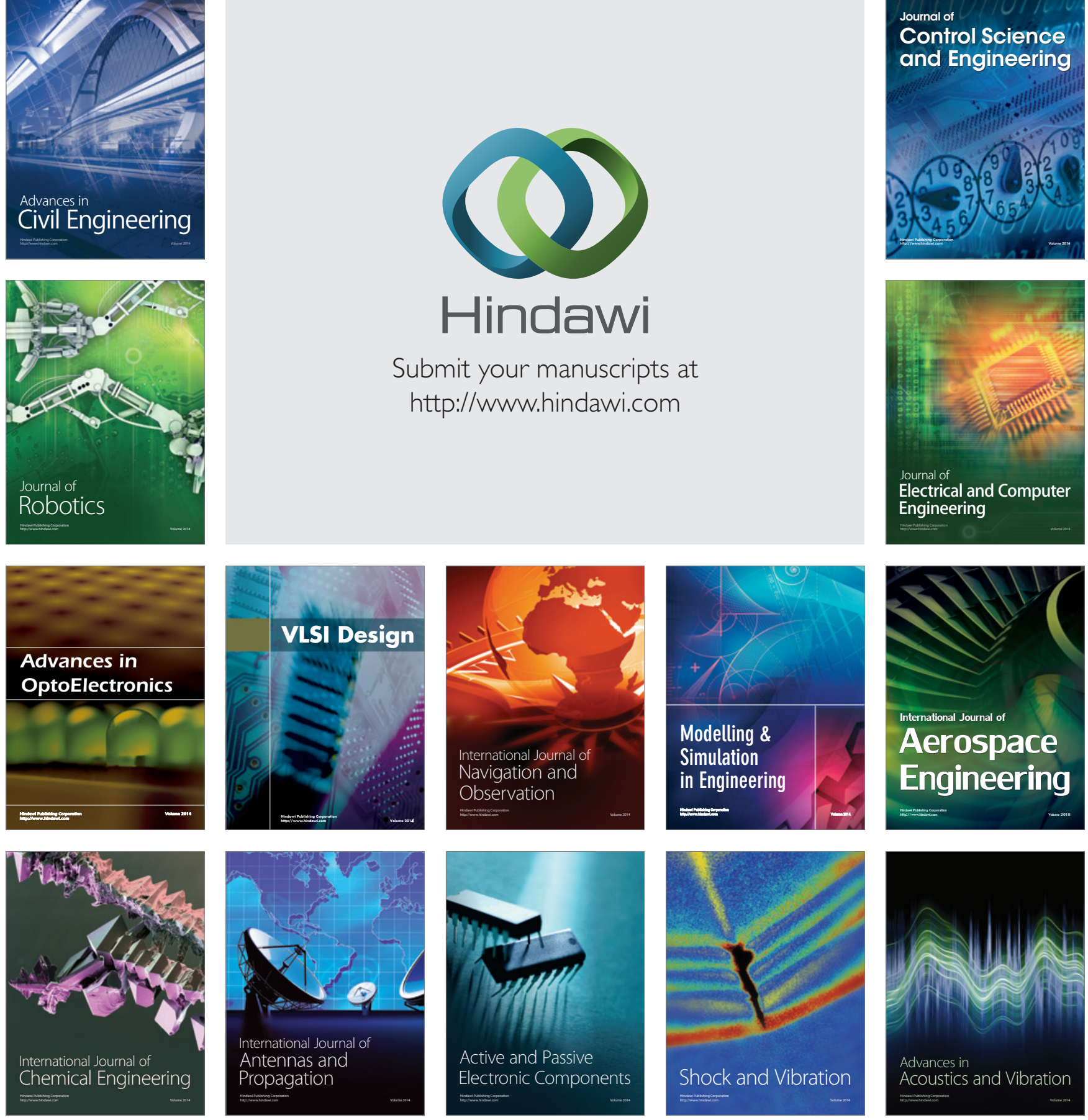